\title{
A Methodology for Tunning Cascade PI Controllers for Power Electronics Converters
}

\author{
Nicolás Muñoz Galeano \\ Grupo GIMEL, Departamento de Ingeniería \\ Eléctrica, Universidad de Antioquia Calle 70 \\ No 52-21, Medellin 050010, Colombia,
}

\author{
Jesús María López Lezama \\ Grupo GIMEL, Departamento de Ingeniería \\ Eléctrica, Universidad de Antioquia Calle 70 \\ No 52-21, Medellín 050010, Colombia,
}

\author{
Juan Bernardo Cano Quintero \\ Grupo GIMEL, Departamento de Ingeniería \\ Eléctrica, Universidad de Antioquia Calle 70 \\ No 52-21, Medellín 050010, Colombia
}

\begin{abstract}
This paper presents a methodology for tuning cascade PI controllers for power electronics (PE) converters. In general terms, this paper presents a step by step mathematical procedure for obtaining the transfer functions useful for tunning PI controllers. Most of converters exhibit nonminimum phase behavior for voltage to duty transfer function; in consequence, cascade PI controller is needed. The proposed cascade PI controller features inner and outer loops. The inner loop allows controlling the inductor current while the outer loop permits to control the capacitor voltage and provides the reference current of the inner loop. The methodology was applied to the Boost converter. Controllers were tuned using Matlab R2020b while control implementation was performed by using Open Modellica.
\end{abstract}

Keywords: Cascade PI controllers, Power Electronics (PE) devices, Transfer functions, Matlab, OpenModelica.

\section{INTRODUCTION}

The design of control structure for Power Electronics (PE) devices is an important topic that concerns electrical, electronic and control engineers. In consequence, there is a great quantity of papers reported in the technical literature regarding this issue; nonetheless, most of these papers are hard to read and understand for undergraduate students. Therefore, this paper presents a step-by-step methodology for tunning cascade PI controllers in PE devices, starting from basic knowledge and reaching more elaborate or complex knowledge useful in the design stage [1]-[8].

The methodology was organized taking into account a bibliographical review [9]-[14]; so the proposed methodology features the following steps: 1) Obtention of Differential equations that govern the behavior of the converter. 2) Application of average model for only considering average values and disregarding voltage and current ripples; this model is called large-signal average model. 3) Linearization of the system using Jacobian Linearization for evaluating the system behavior for small changes near a fixed operation point; this model is called small signal model. 4) Application of Laplace transform for getting the transfer functions. 5) Evaluation of stability by using pole analysis. 6) Evaluation of non-minimal phase behavior by using zero analysis. 7) Most PE converters exhibit non-minimal phase behavior for voltage to duty transfer function, so a cascade PI controller must be stablished. 8) Deduction of transfer function for tuning the outer loop. 9) Obtention of mean values for stablishing the stationary state. 10) Tunning PI controllers using Matlab R2020b. 11) Implementation of control strategy using OpenModellica. 12) Evaluation of control strategy applying perturbations in the system. This paper includes the dynamical characteristics of the system.

For PE devices, few works that consider dynamical characteristics of the system have been published [15]-[19]. However, in these works, the design problem is presented as an optimization problem. In consequence, a procedure to easily control PE devices is still needed. In this paper, a procedure to easily control PE devices is proposed. In this procedure, neither an optimization process is carried out nor is the control structure fixed. It is important to highlight that zeros location is analyzed, this for stablishing if cascade PI controller is required. In general terms, most converters require a cascade PI controller, so that, the main contribution of this paper is the methodology for getting the transfer functions for tunning cascade PI controllers.

This paper is organized as follows: Section II presents the proposed methodology using the Boost converter. Section III corresponds to the results obtained in OpenModelica and Matlab for validating the proposed methodology. Section IV concludes and highlights the most relevant aspects of this paper.

\section{PROPOSED METHODOLOGY}

The proposed methodology is explained using the Boost converter depicted in Figure 1. $v_{i}$ is the input voltage; $L$ is an inductor; $C$ is a capacitor; $Q$ is a power switch, $D$ is a power diode; and $R$ is the resistor used as a load. The following assumptions have been made: 1) Losses are neglected. 2) voltage drop in power switches is not considered. 3) The passive law of sign is used to obtain voltage and current references of the system that are drawn in red color. 


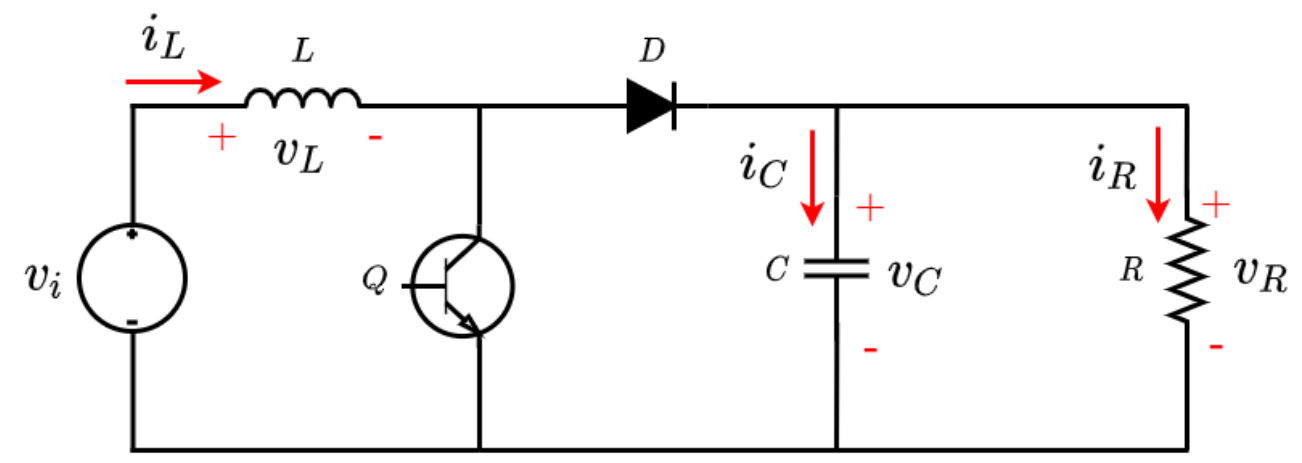

Figure 1. Boost converter topology

Equations (1) and (2) are the differential equations that govern the behavior of the converter and can be obtained if Kirchhoff laws are applied for open and closed switching states. The switching function $u$ is defined to conveniently write the differential equations. When $u=1$, the switch is closed, whereas when $u=0$, the switch is open.

$L \frac{d i_{L}}{d t}=v_{i} \cdot u+\left(v_{i}-v_{C}\right)(1-u)$

$C \frac{d v_{C}}{d t}=-\frac{v_{C}}{R} \cdot u+\left(i_{L}-\frac{v_{C}}{R}\right) \cdot(1-u)$

The average model is applied over Equations (1) and (2). This model only considers the average values of the variables and disregarding their ripples. So, Equations (3) and (4) are also called large-signal average model:

$L \frac{d\left\langle i_{L}\right\rangle}{d t}=\left\langle v_{i}\right\rangle \cdot\langle u\rangle+\left[\left\langle v_{i}\right\rangle-\left\langle v_{C}\right\rangle\right](1-\langle u\rangle)$

$C \frac{d\left\langle v_{C}\right\rangle}{d t}=-\frac{\left\langle v_{C}\right\rangle}{R} \cdot\langle u\rangle+\left[\left\langle i_{L}\right\rangle-\frac{\left\langle v_{C}\right\rangle}{R}\right](1-\langle u\rangle)$

After obtaining the large-signal average model, a linearization of the system is applied. The model linearization or small signal model evaluates the system behavior when small changes near a fixed operation point is applied over variables of the system (Middlebrook, 1988). The following variables are defined: $\left\langle u_{\delta}\right\rangle=\langle u\rangle-\langle\bar{U}\rangle,\left\langle i_{L \delta}\right\rangle=\left\langle i_{L}\right\rangle-\left\langle\overline{I_{L}}\right\rangle,\left\langle v_{i \delta}\right\rangle=$ $\left\langle v_{i}\right\rangle-\left\langle\bar{V}_{l}\right\rangle$, and $\left\langle v_{c \delta}\right\rangle=\left\langle v_{c}\right\rangle-\left\langle\bar{V}_{c}\right\rangle$. Where $\langle u\rangle,\left\langle i_{L}\right\rangle,\left\langle v_{i}\right\rangle,\left\langle v_{c}\right\rangle$ represent the actual values of the converter. $\langle\bar{U}\rangle,\left\langle\bar{I}_{L}\right\rangle,\left\langle\bar{V}_{l}\right\rangle$ and $\left\langle\bar{V}_{c}\right\rangle$ represent their operation point fixed values and $\left\langle u_{\delta}\right\rangle,\left\langle i_{L \delta}\right\rangle,\left\langle v_{i \delta}\right\rangle,\left\langle v_{c \delta}\right\rangle$ represent their small signal deviations.

A small-signal model predicts the system behavior when variables change slightly from its operation point and can be obtained by using the Jacobian linearization (Urrea-Quintero, et al., 2018). Jacobian linearization performs the partial derivatives of the system for obtaining linear relationships in the operation point among all their variables. The Jacobian linearization is expressed as follows:

$\frac{d x}{d t}=f\left(x, x_{1}, x_{2}, \ldots x_{n}\right)$

Its linear expression is as indicated in Equation (6):

$\frac{d x_{\delta}}{d t} \approx A x_{\delta}+A_{1} x_{1_{\delta}}+\cdots+A_{n} x_{n_{\delta}}$

Where:

$$
\begin{aligned}
& A=\left.\frac{\partial f}{\partial x}\right|_{x=\bar{X}, x_{1}=\overline{X_{1}} \ldots x_{n}=\overline{X_{n}}} \\
& A_{1}=\left.\frac{\partial f}{\partial x_{1}}\right|_{x=\bar{X}, x_{1}=\overline{X_{1}} \ldots x_{n}=\overline{X_{n}}} \cdots \\
& A_{n}=\left.\frac{\partial f}{\partial x_{n}}\right|_{x=\bar{X}, x_{1}=\overline{X_{1}} \ldots x_{n}=\overline{X_{n}}}
\end{aligned}
$$

In Equations (5), (6) and (7), $\bar{X}$ represents the variable's operation point and $x_{\delta}$ represent slight deviations from the operation point. A linearization process is applied to Equation (3); initially, it is rewritten as equation (8). Then, the partial derivatives are calculated and evaluated in the operation point (equations (9), (10) and (11)). Finally, the linearized expression is found in equation (12):

$$
\begin{aligned}
\frac{d\left\langle i_{L}\right\rangle}{d t} & =f\left(\left\langle v_{i}\right\rangle,\left\langle v_{C}\right\rangle,\langle u\rangle\right) \\
& =\frac{\left\langle v_{i}\right\rangle \cdot\langle u\rangle+\left[\left\langle v_{i}\right\rangle-\left\langle v_{C}\right\rangle\right](1-\langle u\rangle)}{L}
\end{aligned}
$$


$\frac{\partial f}{\partial\left\langle v_{i}\right\rangle}=\frac{\langle u\rangle+(1-\langle u\rangle)}{L}=\frac{1}{L}$
$\left.\rightarrow \frac{\partial f}{\partial\left\langle v_{i}\right\rangle}\right|_{\left\langle v_{i}\right\rangle=\left\langle\bar{V}_{l}\right\rangle,\left\langle v_{C}\right\rangle=\left\langle\overline{V_{C}}\right\rangle,\langle u\rangle=\langle\bar{U}\rangle}=\frac{1}{L}$

$\frac{\partial f}{\partial\left\langle v_{c}\right\rangle}=\frac{-(1-\langle u\rangle)}{L}$
$\left.\rightarrow \frac{\partial f}{\partial\left\langle v_{c}\right\rangle}\right|_{\left\langle v_{i}\right\rangle=\left\langle\bar{V}_{l}\right\rangle,\left\langle v_{C}\right\rangle=\left\langle\overline{V_{c}}\right\rangle,\langle u\rangle=\langle\bar{U}\rangle}=-\frac{1-\langle\bar{U}\rangle}{L}$

$\frac{\partial f}{\partial\langle u\rangle}=\frac{\left\langle v_{i}\right\rangle-\left[\left\langle v_{i}\right\rangle-\left\langle v_{C}\right\rangle\right]}{L}$

$\left.\rightarrow \frac{\partial f}{\partial\left\langle v_{c}\right\rangle}\right|_{\left\langle v_{i}\right\rangle=\left\langle\overline{V_{l}}\right\rangle,\left\langle v_{C}\right\rangle=\left\langle\overline{V_{c}}\right\rangle,\langle u\rangle=\langle\bar{U}\rangle}=-\frac{\left\langle\overline{V_{c}}\right\rangle}{L}$

$\frac{d\left\langle i_{L \delta}\right\rangle}{d t}=\frac{1}{L}\left\langle v_{i \delta}\right\rangle-\frac{1-\langle\bar{U}\rangle}{L}\left\langle v_{c \delta}\right\rangle+\frac{\left\langle\bar{V}_{c}\right\rangle}{L}\left\langle u_{\delta}\right\rangle$

Analogously, applying the linearization method to Equation (4) yields:

$\frac{d\left\langle v_{C \delta}\right\rangle}{d t}=-\frac{1}{R C}\left\langle v_{c \delta}\right\rangle+\frac{1-\langle\bar{U}\rangle}{C}\left\langle i_{L \delta}\right\rangle-\frac{\left\langle\overline{I_{L}}\right\rangle}{C}\left\langle u_{\delta}\right\rangle$

Where $\langle\bar{U}\rangle,\left\langle\bar{I}_{L}\right\rangle,\left\langle\bar{V}_{l}\right\rangle$ and $\left\langle\bar{V}_{c}\right\rangle$ are constants that depend on the fixed operation point. Equations (12) and (13) correspond to a linear system. Applying Laplace transform to Equations (12) and (13) yields:

$s\left\langle i_{L \delta}\right\rangle=\frac{1}{L}\left\langle v_{i \delta}\right\rangle-\frac{1-\langle\bar{U}\rangle}{L}\left\langle v_{c \delta}\right\rangle+\frac{\left\langle\bar{V}_{c}\right\rangle}{L}\left\langle u_{\delta}\right\rangle$

$s\left\langle v_{C \delta}\right\rangle=-\frac{1}{R C}\left\langle v_{c \delta}\right\rangle+\frac{1-\langle\bar{U}\rangle}{C}\left\langle i_{L \delta}\right\rangle-\frac{\left\langle\overline{I_{L}}\right\rangle}{C}\left\langle u_{\delta}\right\rangle$

The Boost converter model has two input variables: Duty cycle and input voltage. The outputs are the variable states and are defined as the inductor current and capacitor voltage. This paper focuses on obtaining transfer functions related to duty cycle, since the duty cycle is the control variable. Also, a constant input voltage is assumed $\left(\left\langle v_{i}\right\rangle=\right.$ constant $=\left\langle\bar{V}_{l}\right\rangle \rightarrow$ $\left.\left\langle v_{i \delta}\right\rangle=0\right)$, this implies that there are no deviations from its operation point. The solution of $\left\langle v_{C \delta}\right\rangle$ is:

$\left\langle v_{c \delta}\right\rangle=\frac{\frac{1-\langle\bar{U}\rangle}{C}\left\langle i_{L \delta}\right\rangle-\frac{\left\langle\bar{I}_{L}\right\rangle}{C}\left\langle u_{\delta}\right\rangle}{s+\frac{1}{R C}}$
Replacing Equations (16) in (14):

$s\left\langle i_{L \delta}\right\rangle=-\frac{1-\langle\bar{U}\rangle}{L}\left[\frac{\frac{1-\langle\bar{U}\rangle}{C}\left\langle i_{L \delta}\right\rangle-\frac{\left\langle\bar{I}_{L}\right\rangle}{C}\left\langle u_{\delta}\right\rangle}{s+\frac{1}{R C}}\right]+\frac{\left\langle\bar{V}_{c}\right\rangle}{L}\left\langle u_{\delta}\right\rangle$

Rewriting (17) in the form of a transfer function:

$H i(s)=\frac{\left\langle i_{L \delta}\right\rangle}{\left\langle u_{\delta}\right\rangle}=\frac{\left\langle\bar{V}_{c}\right\rangle R C s+\left[\left\langle\bar{V}_{c}\right\rangle+R\left\langle\bar{I}_{L}\right\rangle(1-\langle\bar{U}\rangle)\right]}{R L C s^{2}+L s+R[1-\langle\bar{U}\rangle]^{2}}$

Similarly, transfer function for capacitor voltage against duty cycle is obtained as follows:

$H v(s)=\frac{\left\langle v_{c \delta}\right\rangle}{\left\langle u_{\delta}\right\rangle}=\frac{-\left\langle\bar{I}_{L}\right\rangle R L s+R\left\langle\bar{V}_{c}\right\rangle[1-\langle\bar{U}\rangle]}{R L C s^{2}+L s+R[1-\langle\bar{U}\rangle]^{2}}$

Equations (18) and (19) require pole analysis for determining if the system is stable. Basically, if poles are in the left half of the $s$ plane, the system is stable. Both $\mathrm{Hi}(s)$ and $\mathrm{Hv}(s)$ have the following pair of poles:

$P_{1}, P_{2}=-\frac{1}{2 R C} \pm j \sqrt{\frac{[1-\langle\bar{U}\rangle]^{2}}{L C}-\frac{1}{4 R^{2} C^{2}}}$

From (21), it can be concluded that the Boost converter is stable since the poles are in the left half of the $s$ plane.

A zero analysis is required for determining if the transfer function has non-minimum phase behavior. If zeros are in the left half of the $s$ plane, the control strategy can be implemented following the classical PI loop controller; otherwise, a cascade PI controller must be implemented for avoiding the non-minimum phase behavior. Equation (21) yields zero location for current transfer function $\operatorname{Hi}(s)\left(Z_{1}\right)$, while equation (22) yields zero location for voltage transfer function $H v(s)\left(Z_{2}\right)$.

$Z_{1}=-\frac{\left[\frac{\left\langle\bar{V}_{c}\right\rangle}{R}+\left\langle\bar{I}_{L}\right\rangle(1-\langle\bar{U}\rangle)\right]}{\left\langle\bar{V}_{c}\right\rangle C}$

$Z_{2}=\frac{\left\langle\bar{V}_{c}\right\rangle[1-\langle\bar{U}\rangle]}{\left\langle\overline{I_{L}}\right\rangle}$

Note that $Z_{1}$ is always negative, so the inductor current can be directly controlled using a classical PI controller; however, $Z_{2}$ is always positive, in consequence, the implementation of a cascade PI controller is necessary. Most PE converters require regulation of the voltage capacitor, this paper proposes the cascade PI controller depicted in Figure 2. 


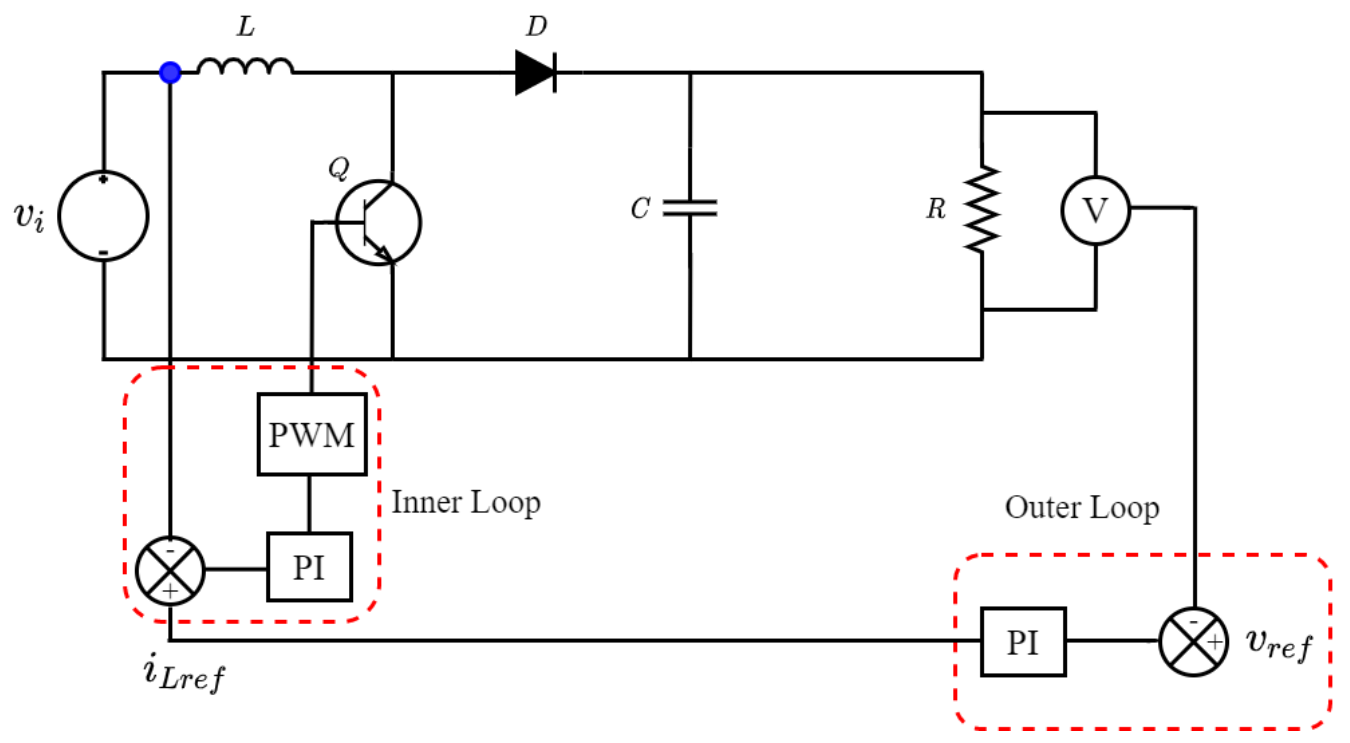

Figure 2. Cascade PI controller

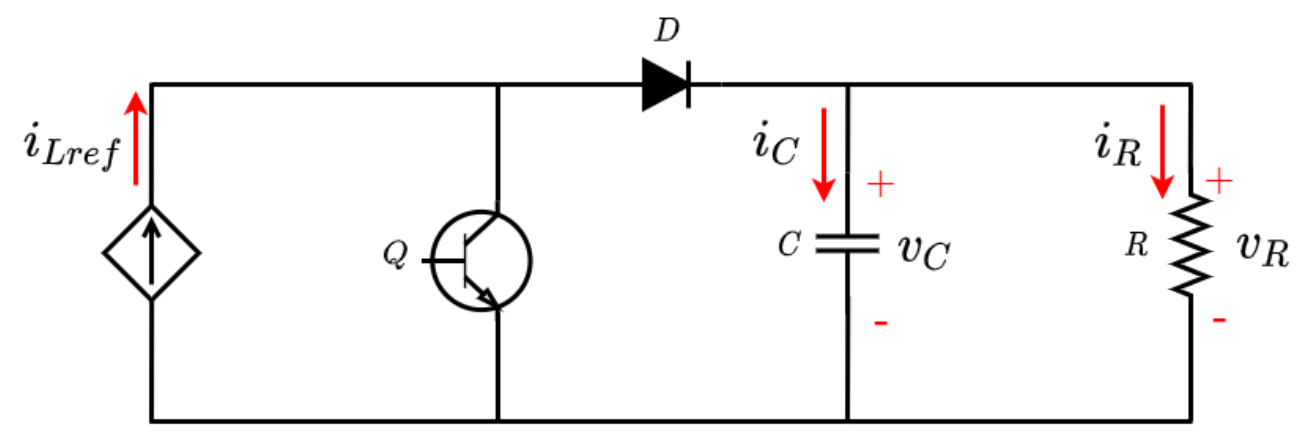

Figure 3. Representation of the Boost converter when the inductor current is controlled

The proposed cascade PI controller has a current inner loop and a voltage outer loop. Setting a voltage reference $\left(v_{r e f}\right)$ for the output voltage, the outer loop gives the inductor reference current $\left(i_{\text {Lref }}\right)$ for the inner loop while the inner loop provides the duty cycle for the Pulse Width Modulation (PWM). For implementing the cascade PI controller, a transfer function is needed for tunning the outer loop. Such transfer function is obtained if it is assumed that the inductor current of the inner loop is controlled. Figure 3 corresponds to the graphical representation of the Boost converter under this condition.

Equation (23) corresponds to the differential equation that was obtained after the application of current Kirchhoff law for both switching states.

$C \frac{d v_{C}}{d t}=i_{\text {Lref }} \cdot(1-u)-\frac{v_{C}}{R}$

The average model is applied on equation (23), which yields:

$C \frac{d\left\langle v_{C}\right\rangle}{d t}=\left\langle i_{\text {Lref }}\right\rangle \cdot(1-\langle\bar{U}\rangle)-\frac{\left\langle v_{C}\right\rangle}{R}$
The small-signal model is obtained taking into account that: $\left\langle v_{c \delta}\right\rangle=\left\langle v_{c}\right\rangle-\left\langle\bar{V}_{c}\right\rangle,\left\langle u_{\delta}\right\rangle=\langle u\rangle-\langle\bar{U}\rangle, \quad$ and $\left\langle i_{\text {Lref } \delta}\right\rangle=$ $\left\langle i_{\text {Lref }}\right\rangle-\left\langle\overline{I_{\text {Lref }}}\right\rangle$. Where $i_{\text {Lref }}$ is the actual reference current, $\overline{I_{L r e f}}$ is the average value of the reference current in the operation point, and $i_{\text {Lref } \delta}$ is its corresponding small deviation. The procedure for obtaining the transfer function (Equation (30)) is as follows:

$\frac{d\left\langle v_{c}\right\rangle}{d t}=f\left(\left\langle i_{\text {Lref }}\right\rangle,\left\langle v_{C}\right\rangle\right)=\frac{\left\langle i_{\text {Lref }}\right\rangle \cdot(1-\langle\bar{U}\rangle)-\frac{\left\langle v_{C}\right\rangle}{R}}{C}$

$\left.\frac{\partial f}{\partial\left\langle i_{\text {Lref }}\right\rangle}\right|_{\left\langle v_{C}\right\rangle=\left\langle\overline{V_{C}}\right\rangle,\left\langle i_{\text {Lref }}\right\rangle=\left\langle\overline{I_{\text {Lref }}}\right\rangle}=\frac{1-\langle\bar{U}\rangle}{C}$

$\left.\frac{\partial f}{\partial\left\langle v_{C}\right\rangle}\right|_{\left\langle v_{C}\right\rangle=\left\langle\overline{V_{C}}\right\rangle,\left\langle i_{\text {Lref }}\right\rangle=\left\langle\overline{\left.I_{\text {Lref }}\right\rangle}\right.}=-\frac{1}{R C}$

$\frac{d\left\langle v_{C \delta}\right\rangle}{d t}=-\frac{1}{R C}\left\langle v_{C \delta}\right\rangle+\frac{1-\langle\bar{U}\rangle}{C}\left\langle i_{\text {Lref } \delta}\right\rangle$ 
International Journal of Engineering Research and Technology. ISSN 0974-3154, Volume 13, Number 11 (2020), pp. 3997-4003

(C) International Research Publication House. https://dx.doi.org/10.37624/IJERT/13.11.2020.3997-4003

$\mathrm{s}\left\langle v_{C \delta}\right\rangle=-\frac{\left\langle v_{C \delta}\right\rangle}{R C}+\frac{1-\langle\bar{U}\rangle}{C}\left\langle i_{L r e f \delta}\right\rangle$

$\frac{\left\langle v_{C \delta}\right\rangle}{\left\langle i_{\text {Lref } \delta}\right\rangle}=\frac{(1-\langle\bar{U}\rangle) R}{R C S+1}$

Note that transfer function of Equation (30) is stable and does not exhibit the non-minimal phase behavior.

The mean values required in transfer functions can be obtained if Equations (31) and (32) are equal to zero:

$\left\langle\bar{V}_{c}\right\rangle=\frac{\left\langle\bar{V}_{l}\right\rangle}{1-\langle\bar{U}\rangle}$

$\left\langle\bar{I}_{L}\right\rangle=\frac{\left\langle\bar{V}_{c}\right\rangle}{R[1-\langle\bar{U}\rangle]}$

\section{RESULTS}

A simulation was carried out using the default compiler and solver in OpenModelica Connection Editor (OMEdit), version 3.2.2. Figure 4 shows the implementation in OpenModelica of the converter depicted in Figure 1. The switching frequency is $20 \mathrm{kHz}$ and the setpoint of the output voltage is $40 \mathrm{~V}$. The Boost converter was parametrized as follows: $L=10 \mathrm{mH}$, $C=2000 \mu F, R=10 \Omega, v_{i}=20 \mathrm{~V}$. The values of the PI controllers were obtained using Sisotool (Matlab R2020b). The inner loop parameters were obtained using Equation (19) while the outer loop parameters were obtained using Equation (31). The inner loop has a gain of 0.06 and a time constant of $0.055 \mathrm{~s}$ while outer loop has a gain of 0.2751 and a time constant of $0.05 \mathrm{~s}$. The PI controllers were design considering a damping factor of 0.707 . Also, the inner loop bandwidth must be smaller than switching frequency while outer loop bandwidth must be smaller than the inner loop bandwidth.

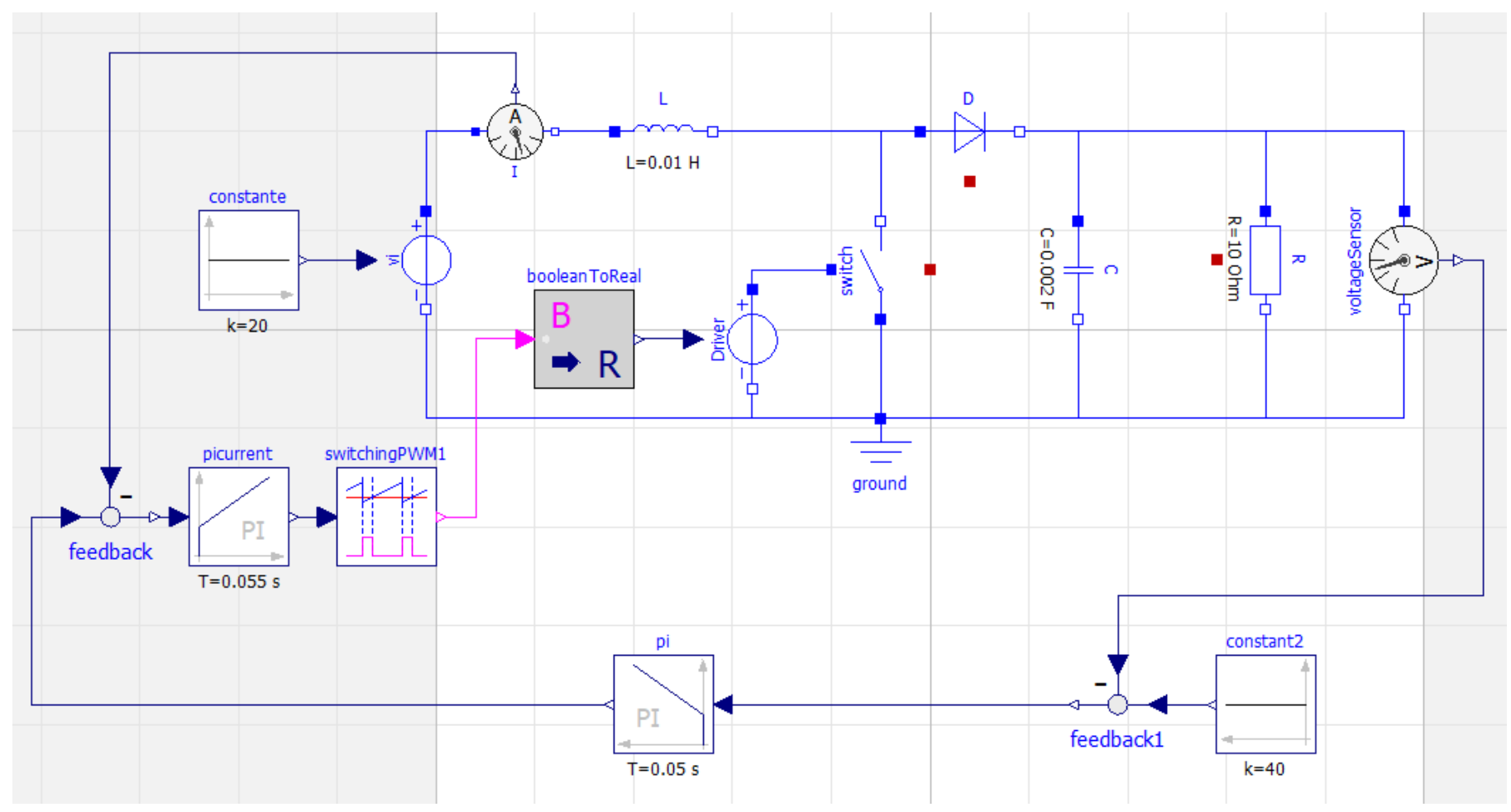

Figure 4. Implementation in OpenModelica.

Figure 5 shows in red color the output voltage while in blue color the input current. In $\mathrm{t}=0$ the system successfully starts following the output voltage $(40 \mathrm{~V})$, not presenting overshoots. In $\mathrm{t}=1.5 \mathrm{~s}$, the input voltage was increased to $25 \mathrm{~V}$. In $\mathrm{t}=3$ $\mathrm{seg}$, the load resistance was reduced to $5 \Omega$. In $\mathrm{t}=4.5 \mathrm{~s}$, the input voltage was decreased to $20 \mathrm{~V}$. For all perturbations and changes, the system follows the reference, keeping the output voltage in $40 \mathrm{~V}$; however current changes for guarantying the balance of power of the system. 


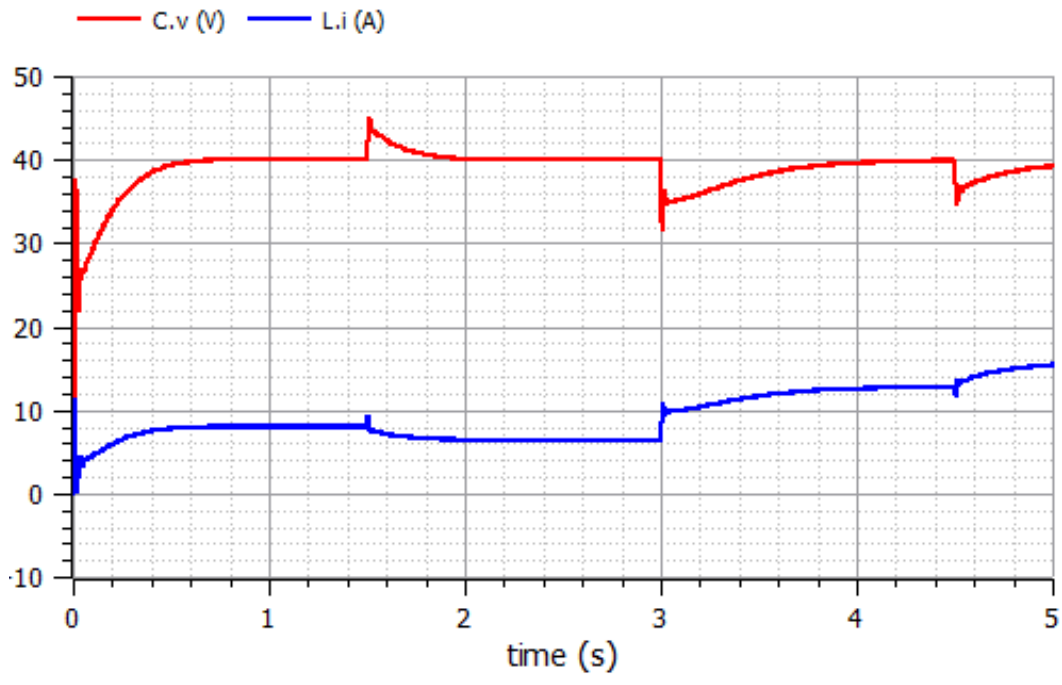

Figure 4. Voltage and current waveforms under perturbations

\section{CONCLUSIONS}

This paper presented a methodology for tunning cascade PI controllers in PE converters. The methodology included the procedure for obtaining the transfer functions giving specific details in the deduction of equations. The implemented cascade PI controller has an inner and an outer loop. The inner loop controls the inductor current while the outer loop controls the output voltage. Controllers were tuned using Sisotool (Matlab), the inner loop was tuned and posteriorly the outer loop was tuned assuming that inner loop was controlled. The control implementation was done in OpenModellica. Under perturbations in input voltage and load resistor the converter successfully follows the output voltage which validates the proposed methodology and the deduced transfer functions.

\section{ACKNOWLEDGEMENTS}

The authors gratefully acknowledge the financial support provided by the Colombia Scientific Program within the framework of the call Ecosistema Científico (Contract No. FP44842- 218-2018). Likewise, Universidad de Antioquia (Colombia) is acknowledged for the financial support through the Sostenibilidad program.

\section{REFERENCES}

[1] Arango, E., Ramos-Paja, C. A., Calvente, J., Giral, R., and Serna-Garces, S. I. (2013). Asymmetrical Interleaved DC/DC Switching Converters for Photovoltaic and Fuel Cell Applications - Part 2: Control-Oriented Models. Energies, 6(10), 5570-5596.

[2] Bi, Z. and Xia, W. (2010). Modeling and Simulation of Dual-Mode DC/DC Buck Converter. Second International Conference on Computer Modeling and Simulation, Sanya, Hainan (China), 371-375.
[3] Urrea-Quintero, JH., Muñoz-Galeano, N., GómezEchavarría, LM. (2018). Analysis and Control of Power Electronic Converters Based on a System Zeros Location Approach. In Anh Tuan, L. Applied Modern Control (pp.1-22). London: Intechopen.

[4] Duong, T-D., Nguyen, M-K., Tran, T-T., Lim, Y-C., Choi, J-H. (2019). Transformerless High Step-Up DCDC Converters with Switched-Capacitor Network. Electronics, 8(12), 1420.

[5] Martínez-García, MS., de Castro, A., Sanchez, A., Garrido, J. (2019). Analysis of Resolution in Feedback Signals for Hardware-in-the-Loop Models of Power Converters. Electronics, 8(12), 1527.

[6] Shaw, P. (2019). Modelling and analysis of an analogue MPPT-based PV battery charging system utilising dc-dc boost converter. IET Renewable Power Generation, 13(11), 1958-1967

[7] Velilla, E., Cano, J. B., Jaramillo, F. (2019). Monitoring system to evaluate the outdoor performance of solar devices considering the power rating conditions. Solar Energy, 194(1), 79-85

[8] Yang, T., Liao, Y. Discrete Sliding Mode Control Strategy for Start-Up and Steady-State of Boost Converter. (2019). Energies, 12(15), 2990.

[9] Liu, J., Hu, J. and Xu, L. (2007). Dynamic Modeling and Analysis of Z Source Converter -- Derivation of AC Small Signal Model and Design-Oriented Analysis. Power Electron. IEEE Trans., 22(5), 1786-1796.

[10] Beldjajev, V. and Roasto, I. (2012). Efficiency and Voltage Characteristics of the Bi-Directional Current Doubler Rectifier. Przeglad Elektrotechniczny, 88(8), 124-129.

[11] Rouzbehi, K., Miranian, A., Escaño, JM., Rakhshani, E., Shariati, N., Pouresmaeil, E. (2019). A Data-Driven Based Voltage Control Strategy for DC-DC 
Converters: Application to DC Microgrid. Electronics, 8(5), 493.

[12] Davoudi, A., Jatskevich, J., Chapman, P. L. and Bidram, A. (2013). Multi-Resolution Modeling of Power Electronics Circuits Using Model-Order Reduction Techniques. IEEE Transactions on Circuits and Systems I: Regular Papers, 60(3), 810-823.

[13] Geyer, T., Papafotiou, G., Frasca, R. and Morari, M. (2008). Constrained Optimal Control of the Step-Down DC-DC Converter. IEEE Transactions on Power Electronics, 23(5), 2454-2464.

Liang, T. J. and Tseng, K. C. (2005). Analysis of integrated boost-flyback step-up converter. IEE Proceedings - Electric Power Applications, 152(2), 217-225.

[14] Restrepo, C., Konjedic, T., Calvente, J., Milanovic, M. and Giral, R. (2013). Fast Transitions Between Current Control Loops of the Coupled-Inductor Buck-Boost DC-DC Switching Converter. IEEE Transactions on Power Electronics, 28(8), 3648-3652.

[15] Fritzson, P., Pop, A., Asghar, A., Bachmann, B., Braun, W., Braun, R., ... , Franke, R. (october, 2019). The OpenModelica Integrated Modeling, Simulation, and Optimization Environment. In Proceedings of The American Modelica Conference 2018, Somberg Conference Center, Cambridge MA, USA, Linköping University Electronic Press.

[16] Dizqah, A. M., Maheri, A., Busawon, K., Fritzson, P. (2015). Standalone DC microgrids as complementarity dynamical systems: Modeling and applications. Control Engineering Practice, 35, 102-112.

[17] Bartolini, A., Casella, F., Guironnet, A. (march, 2019). Towards Pan-European Power Grid Modelling in Modelica: Design Principles and a Prototype for a Reference Power System Library. In Proceedings of the 13th International Modelica Conference, Regensburg (Germany), Linköping University Electronic Press.

[18] Reid, D. (april, 2015). DQ rotating frame PI control algorithm for power inverter voltage regulation modelling and simulation using the OpenModelica platform. In SoutheastCon 2015, Fort Lauderdale (EEUU), IEEE.

[19] Murad, M. A. A., Vanfretti, L., Rokonuzzaman, M., Tuhin, R. A. (september, 2017). Enhancing engineering studies in developing countries using OpenModelica. In 2017 4th International Conference on Advances in Electrical Engineering, Dhaka (Bangladesh) 